\title{
Motivational salience modulates hippocampal repetition suppression and functional connectivity in humans
}

\author{
Sarah Zweynert ${ }^{1,2}$, Jan Philipp Pade ${ }^{1,2}$, Torsten Wüstenberg ${ }^{2}$, Philipp Sterzer $^{2}$, Henrik Walter ${ }^{2}$, \\ Constanze I. Seidenbecher ${ }^{1}$, Alan Richardson-Klavehn ${ }^{3}$, Emrah Düzel ${ }^{3,4,5}$ and Björn Hendrik Schott ${ }^{1,2,3}{ }^{*}$ \\ 1 Leibniz Institute for Neurobiology, Magdeburg, Germany \\ ${ }^{2}$ Department of Psychiatry, Campus Mitte, Charité University Hospital, Berlin, Germany \\ ${ }^{3}$ Department of Neurology, Otto von Guericke University, Magdeburg, Germany \\ ${ }^{4}$ Institute for Cognitive Neurology and Dementia Research, Otto von Guericke University, Magdeburg, Germany \\ ${ }^{5}$ Institute for Cognitive Neuroscience, University College London, London, UK
}

\section{Edited by:}

Neal J. Cohen, University of Illinois, USA

\section{Reviewed by:}

Bradford C. Dickerson, Harvard

Medical School, USA

Shu-Chen Li, Max Planck Institute for

Human Development, Germany

\section{*Correspondence:}

Björn Hendrik Schott, Leibniz Institute for Neurobiology, Brenneckestr. 6,

39118 Magdeburg, Germany.

e-mail: bschott@neuro2.med.

uni-magdeburg.de
Repetition suppression (RS) is a rapid decrease of stimulus-related neuronal responses upon repeated presentation of a stimulus. Previous studies have demonstrated that negative emotional salience of stimuli enhances RS. It is, however, unclear how motivational salience of stimuli, such as reward-predicting value, influences RS for complex visual stimuli, and which brain regions might show differences in RS for reward-predicting and neutral stimuli. Here we investigated the influence of motivational salience on RS of complex scenes using event-related functional magnetic resonance imaging. Thirty young healthy volunteers performed a monetary incentive delay task with complex scenes (indoor vs. outdoor) serving as neutral or reward-predicting cue pictures. Each cue picture was presented three times. In line with previous findings, reward anticipation was associated with activations in the ventral striatum, midbrain, and orbitofrontal cortex (OFC). Stimulus repetition was associated with pronounced RS in ventral visual stream areas like the parahippocampal place area (PPA). An interaction of reward anticipation and RS was specifically observed in the anterior hippocampus, where a response decrease across repetitions was observed for the reward-predicting scenes only. Functional connectivity analysis further revealed specific activity-dependent connectivity increases of the hippocampus and the PPA and OFC. Our results suggest that hippocampal RS is sensitive to reward-predicting properties of stimuli and might therefore reflect a rapid, adaptive neural response mechanism for motivationally salient information.

Keywords: reward, repetition suppression, priming, hippocampus, fMRI

\section{INTRODUCTION}

Stimulus repetition is commonly associated with an initially strong neural response to a stimulus, followed by rapidly decreasing responses to repeated presentation of the same stimulus, phenomenon known as repetition suppression (RS; Desimone, 1996; Ishai et al., 2004) or repetition decrease (Vuilleumier et al., 2005). RS occurs in secondary visual and stimulus-related cortical areas as well as prefrontal association cortices. In the hippocampus and adjacent medial temporal lobe (MTL) structures, RS is particularly pronounced, especially after short delays (Brozinsky et al., 2005), and there is converging evidence that the hippocampus acts as a detector of novel information (Tulving et al., 1996; Ranganath and Rainer, 2003).

Several functional magnetic resonance imaging (fMRI) studies have demonstrated that RS can be modulated by attention, expectancy, or negative emotional salience (Ishai et al., 2004; Vuilleumier et al., 2005; Summerfield et al., 2008). Both attended and emotionally salient visual stimuli show stronger RS in stimulus-related brain regions when compared to unattended or neutral stimuli, respectively. RS can potentially arise from at least three distinct neural mechanisms: fatigue, response sharpening, and response facilitation (Grill-Spector et al., 2006). According to the sharpening model (Desimone, 1996), cortical stimulus representations become more specific by recruiting a decreasing number of neurons when a stimulus is repeated. The facilitation model implies that RS results from more rapid processing of repeated stimuli (James and Gauthier, 2006). A network model of cortical responses (Friston, 2005) further suggests that neural response patterns of stimulus-sensitive cortical structures code a prediction error that is compared to previously existing stimulus representations in modulatory brain structures and decreases with increasing stimulus familiarity. Such models are in line with the notion that $\mathrm{RS}$ is particularly pronounced for salient and behaviorally relevant stimuli.

Reward-predicting properties of stimuli are particularly salient, and they are rapidly encoded by the reward system of the ventral striatum (VS)/nucleus accumbens (NAcc). Unexpected reward elicits dopamine release in the NAcc by afferences from the substantia nigra/ventral tegmental area (SN/VTA), and stimulus-reward association learning leads to a temporal shift of this response to the reward-predicting stimulus (Schultz, 1997; McClure et al., 2003). There is a close relationship between the 
mesolimbic reward system and hippocampus-dependent memory function. Reward-predicting stimuli are more likely to be explicitly remembered (Wittmann et al., 2005), and enhanced dopaminergic activity is also elicited by stimulus novelty (Düzel et al., 2010). Lisman and Grace (2005) suggested that hippocampal novelty signals are conveyed to the NAcc, triggering increased hippocampal dopamine release, which in turn promotes long-term encoding.

Despite well-documented interactions of reward processing and hippocampus-dependent memory and influences of salience on RS, the effects of reward-predicting stimulus properties on RS are yet unclear. Here we investigated how RS is modulated by reward-predicting stimulus features. We hypothesized that RS might be enhanced for reward-predicting stimuli, as previously demonstrated for emotional or attended stimuli (Ishai et al., 2004; Vuilleumier et al., 2005). Because of recent evidence for a common adaptive coding mechanism for novelty and reward in the hippocampus (Bunzeck et al., 2010), we further hypothesized that the hippocampus might act as an interface linking mesolimbic reward prediction and stimulus processing in visual association cortices.

\section{MATERIALS AND METHODS PARTICIPANTS}

Thirty young, healthy adults (16 women, 14 men; mean age 24.6 years, range 19-30 years) volunteered for paid participation in the experiment. All underwent a routine clinical interview for neurological or psychiatric disorders. Exclusion criteria were present or past neurological or psychiatric disease and the use of centrally acting drugs. Participants included 29 right-handed subjects and one ambidextrous person. All participants gave written informed consent to participate in the study, in accordance with the Declaration of Helsinki, and the study was carried out as approved by the local ethics committee.

\section{PARADIGM}

The experiment consisted of two sessions, each comprising a total of 180 trials ( 30 trials per condition: reward vs. neutral $\times 1$ st, 2 nd, and 3rd presentation). Participants were instructed to perform an adapted version of a monetary incentive delay (MID) task (Wittmann et al., 2005) with photographs of indoor and outdoor scenes serving as reward or neutral cues, respectively (counterbalanced across participants; Schott et al., 2008; Krebs et al., 2009a,b). Participants were explicitly informed, which condition (indoor or outdoor) would be rewarded. Each cue picture was presented for $1000 \mathrm{~ms}$. After a variable delay, a one-digit target number was presented for $100 \mathrm{~ms}$, and participants indicated via button press whether this number was greater or smaller than 5 (Wittmann et al., 2005). The ensuing feedback indicated a win for a correct and fast answer, a loss for an incorrect and/or too slow answer or a neutral feedback for a non-rewarded trial. The feedback was presented for $600 \mathrm{~ms}$. Both the delay between the cue picture and the target number, and the inter-stimulus interval varied from 2 to $6 \mathrm{~s}$, using a pseudo-exponential jitter, to optimize estimation of the trial-specific hemodynamic responses (Hinrichs et al., 2000). The trial structure is depicted in Figure 1. Because RS is most reliably observed when lags between repetitions are short (Brozinsky et al., 2005), the paradigm was designed in a way that, over the course of 18 trials, each cue picture was presented three times in a pseudo-random order.

Before entering the MR tomograph, a learning session was performed during which participants performed a short version of the task consisting of each 10 rewarded and neutral trials (using pictures not presented during the actual experiment), in order to minimize effects of stimulus-reward association learning during the scanning phase and to estimate individual reaction time (RT) thresholds for each participant. These thresholds were then used as response deadlines during the actual task in order to obtain a $70-80 \%$ hit rate in the rewarded condition (Wittmann et al., 2005; Schott et al., 2007). The response deadline was about $400 \mathrm{~ms}$ for most participants.

\section{IMAGE ACQUISITION}

T2*-weighted echo-planar images (EPIs) were acquired on a 1.5T whole-body MRI system (GE Signa Horizon, General Electric),

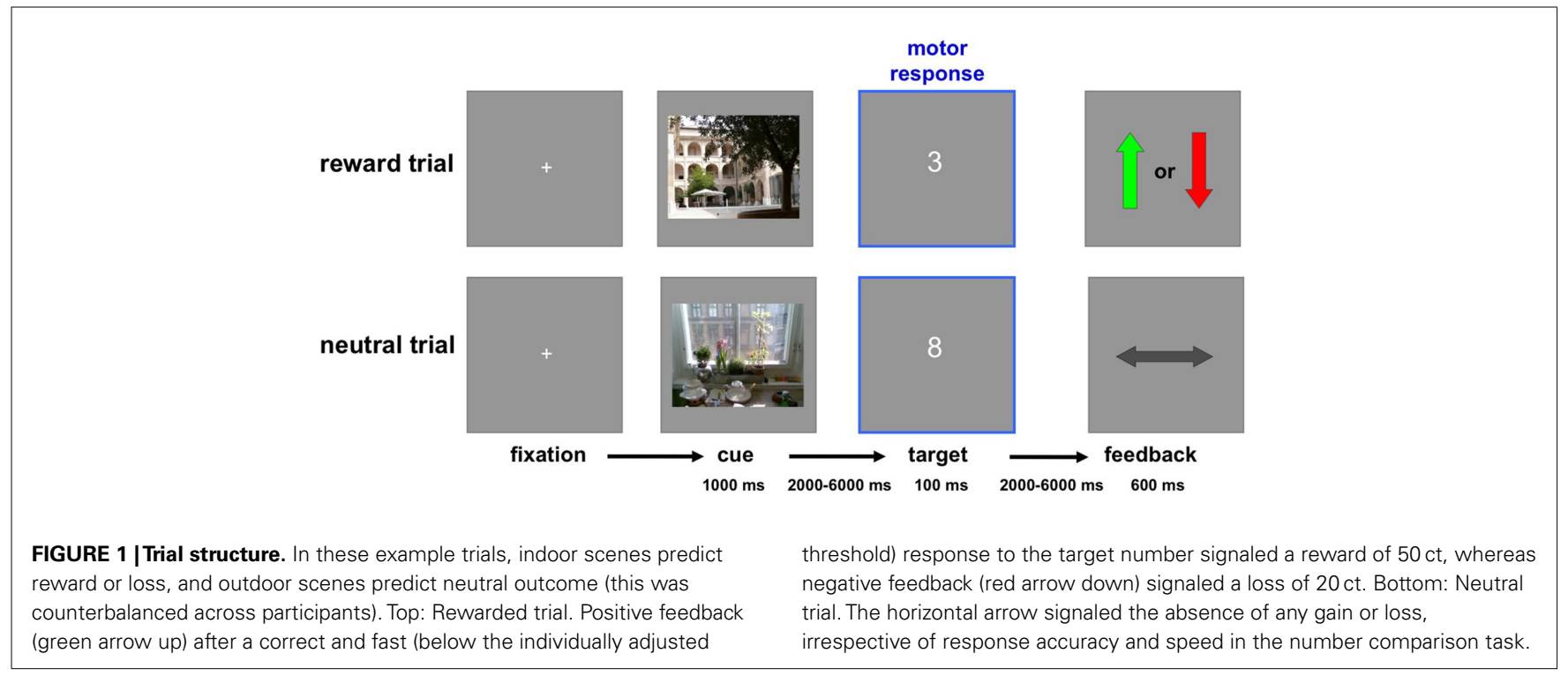


using an eight channel circular polarized head coil. Before functional image acquisition, a T1-weighted 3D fast spoiled gradient echo (FSPGR) image was acquired to allow proper positioning of the functional slices.

T2*-weighted gradient echo echo-planar images (GE-EPIs) comprising 23 slices parallel to the anterior-posterior commissure line as determined from the FSPGR image were acquired in an odd-even interleaved ascending order ( $T R=2000 \mathrm{~ms}$; $\mathrm{TE}=35 \mathrm{~ms}$; matrix size $=64 \times 64$; slice thickness $=4 \mathrm{~mm}$; interslice gap $=1 \mathrm{~mm}$ gap; voxel size $=3.13 \mathrm{~mm} \times 3.13 \mathrm{~mm} \times 5 \mathrm{~mm}$ ). Each of the three scanning sessions comprised 540 volumes. Six additional volumes were acquired at the beginning of each functional session and subsequently discarded from the analysis to allow for steady-state magnetization. To optimize normalization, a co-planar proton density (PD)-weighted image was acquired in the same session as the functional images.

\section{DATA PROCESSING AND ANALYSIS}

Data analysis was performed using Statistical Parametric Mapping (SPM8b; Wellcome Trust Centre for Neuroimaging, London, UK). EPIs were corrected for acquisition delay and head motion. Because of their superior gray-white contrast in subcortical structures such as the NAcc and the SN, PD images were chosen for estimation of normalization parameters (D'Ardenne et al., 2008; Schott et al., 2008). Thus, the co-planar PD image was coregistered to the mean EPI, normalized into a common stereotactic reference frame (ICBM, International Consortium for Brain Mapping, http://www.loni.ucla.edu/ICBM/) and segmented using the segmentation algorithm implemented in SPM8b. Spatial normalization of EPIs was performed using the normalization parameters determined from segmentation. Images were re-sampled to a voxel size of $3 \mathrm{~mm} \times 3 \mathrm{~mm} \times 3 \mathrm{~mm}$ and spatially smoothed using an isotropic Gaussian kernel with $(8 \mathrm{~mm}$ full-width at half maximum). The resulting voxel time series were high-pass filtered with a cut-off frequency of $128 \mathrm{~s}$. Serial correlations from aliased cardiological and respiratory effects were accounted for using a 1st-order autoregressive model.

Statistical analysis was performed in a two-stage mixed-effects model. In the first stage, neural activity was modeled by a delta function at stimulus onset. The ensuing hemodynamic response was modeled by convolving these delta functions with the canonical hemodynamic response function (HRF) provided by SPM. The resulting time courses were down-sampled for each scan to form regressors in a general linear model (GLM).

The GLM contained separate regressors for each condition of interest (1st, 2 nd, and 3rd presentation $\times$ reward vs. neutral cues; positive, negative, and neutral feedback; target numbers), covariates of no interest for the six rigid-body-movement parameters determined from motion correction (to capture signal fluctuations related to the movement $\times$ susceptibility interaction), and a single constant representing the mean over scans. The model was fitted to the data using the restricted maximum likelihood algorithm as implemented in SPM.

At the second stage of statistical analysis, contrasts of the parameter estimates for the conditions of interest were submitted to second level random effects analyses. The two-way ANOVA for repeated measures included the within-subject factors
REPETITON (1st, 2nd, and 3rd) and REWARD (rewarded or non-rewarded). Within this model, T-contrasts of interest were computed (1st vs. 3rd repetition, rewarded vs. non-rewarded cues, positive interaction of rewarded vs. non-rewarded $\times 1$ st vs. 3 rd repetition). A statistical threshold of $p=0.001$, uncorrected was adopted for all comparisons, with a minimum cluster size of 10 adjacent voxels $\left(270 \mathrm{~mm}^{3}\right)$. Small-volume corrections were performed using anatomically defined or literature-based probabilistic regions of interest [ROIs, hippocampus, parahippocampal place area (PPA), VS; see below].

\section{REGION OF INTEREST ANALYSES}

For detailed ROI analysis of medial temporal RS, ROIs delineating the left and right MTL structures (hippocampus, amygdala, and parahippocampal cortex) were obtained from the WFU Pickatlas toolbox for SPM (http://fmri.wfubmc.edu/software/PickAtlas). As a first step of the ROI analyses in MTL, we computed the positive interaction of repetition and reward (i.e., larger RS for rewarded as compared to neutral pictures), thresholded at $p<0.005$, uncorrected. We then computed significance levels and cluster sizes within ROIs of the bilateral hippocampus, parahippocampal cortex, and amygdala. Only MTL activations surviving a smallvolume correction for family wise error (FWE) for the volume of the respective ROIs were considered reliable.

For the PPA and the VS, probabilistic literature-based ROIs were created using a previously described algorithm (Schubert et al., 2008). To this end, we selected the coordinates of PPA and VS activation maxima observed in recent studies (PPA: Peelen and Downing, 2005; Epstein and Higgins, 2007; Epstein et al., 2007; Henson and Mouchlianitis, 2007; Park et al., 2007, 2009; Xu et al., 2007; Henderson et al., 2008; Horner and Andrews, 2009; VS: Knutson et al., 2001a,b; O’Doherty et al., 2004; Wittmann et al., 2005; Abler et al., 2006, 2007; Hariri et al., 2006; Juckel et al., 2006; Scheres et al., 2007; Schott et al., 2007, 2008; Camara et al., 2010). The coordinates of all local maxima of activation reported by the authors were pooled and, if necessary, transformed from Talairach to MNI space, using the affine algorithm proposed by Brett et al. (2001). Based on this data set, we created the ROIs using the following steps:

(1) The probability that a voxel at a given position lay within the area of interest was estimated by calculating a 3D normal (Gaussian) distribution $G(x, y, z)$ as follows (Turkeltaub et al., 2002):

$$
\begin{aligned}
& G(x, y, z)=\frac{1}{2 \pi \sqrt{|\operatorname{Det}(C)|}} \exp \\
& \left(-\frac{1}{2}\left[\begin{array}{lll}
x-\tilde{x} & y-\bar{y} & z-\bar{z}
\end{array}\right] C^{-1}\left[\begin{array}{l}
x-\tilde{x} \\
y-\bar{y} \\
z-\bar{z}
\end{array}\right]\right)
\end{aligned}
$$

where $C$ was the covariance matrix for all coordinate triples $x$, $y, z$ from the underlying literature and $\bar{x}, \bar{y}, \bar{z}$ were the mean values of the $x, y$, and $z$ coordinates, respectively (Nielsen and Hansen, 2002).

(2) Because the resulting distribution also contained voxels located in white matter and extracerebral space, we restricted 
the 3D distribution only to those voxels that belong to gray matter with a probability of at least $50 \%$. To this end we used the gray matter probability map as provided by SPM8.

(3) The outer limits of the finally used ROI were defined by a threshold of $n \mathrm{SD}$ of the resulting 3D distribution. Finally a binary mask including all surviving voxels was formed.

(4) For the VS, the binary mask was further masked inclusively with the anatomical ROI of the striatum obtained from the WFU Pickatlas.

[Note: The script for generating the probabilistic ROIs (written in Matlab by author Torsten Wüstenberg) and the full lists of coordinates used for ROI generation can be obtained from the authors upon request].

\section{FUNCTIONAL CONNECTIVITY ANALYSIS}

To assess possible changes in functional connectivity due to modulation by motivational salience we calculated an additional analysis to uncover psycho-physiological interactions (PPI; Friston et al., 1997; Gitelman et al., 2003; Das et al., 2005). PPI analysis captures the functional coupling between different brain regions in relation to a psychological variable (in the present study: repeated presentation of cue pictures in the rewarded and neutral conditions). Based on the observed RS by reward interaction, we used the right anterior hippocampus as seed region. To extract the individual BOLD data from this region, a binary mask image containing right cornu ammonis (CA) and subiculum was created according the maximum probability map (MPM) approach described by Eickhoff et al. (2006) and as implemented in the probabilistic cyto-architectonical brain atlas for SPM version 1.7 (Eickhoff et al., 2005). Within this mask for each subject, a sphere with a radius of $6 \mathrm{~mm}$ was centered on the voxel with the highest variance explanation for the applied model and first eigenvariate time series (adjusted for experimental effects of interest) from this sphere were extracted and deconvolved with the canonical HRF. The resulting time course was convolved with the psychological functions $P_{\text {reward }}$ and $P_{\text {neutral }}$ of time $t$ (in scans), which was set to 3 for the first, 2 for the second, and 1 for the third presentation of the neutral and cue pictures, respectively, and 0 in all other cases. Reconvolution of the resulting function with the HRF yielded the vectors $X_{\text {reward }}$ and $X_{\text {neutral }}$, which formed the primary covariates of interest in the design matrix of a new GLM. $P_{\text {reward }}$ and $P_{\text {neutral }}$ were also convolved with the HRF to form further covariates. The fifth covariate was the original BOLD eigenvariate. The six rigidbody-movement parameters determined from motion correction and a constant representing the mean over scans were included in the design matrix as covariates of no interest. Model estimation was performed as described above. At second level, a two-way ANOVA for repeated measures was conducted to compare the psycho-physiological interaction contrasts for rewarded and neutral pictures. The significance level of the connectivity analysis was set to $p=0.001$, uncorrected, with a minimum cluster size of 10 adjacent voxels $\left(270 \mathrm{~mm}^{3}\right)$. A small-volume FWE-corrected significance level of 0.05 was applied to the PPA using a probabilistic literature-based ROI as described above.

\section{RESULTS \\ BEHAVIORAL RESULTS}

All participants responded to the target numbers with high accuracy. RTs were longer for neutral relative to rewarded items, leading to lower successful response rates (i.e., correct target responses within the individually adjusted time limit) in the neutral relative to the rewarded condition (Table 1). Furthermore, repetition (1st to 3rd presentation of cue pictures) was associated with a higher successful response rate. A two-way ANOVA for repeated measures on the successful target responses revealed significant main effects of reward $\left(F_{1,29}=65.84, p<0.0001\right)$ and repetition $\left(F_{1.86,54.0}=4.31 ; p=0.020\right.$; Greenhouse-Geissercorrection for non-sphericity applied). RTs showed a significant effect of reward $\left(F_{1,29}=71.79 ; p<0.0001\right.$; two-way ANOVA for repeated measures), and there was a significant reward by repetition interaction $\left(F_{1.73,50.04}=5.27 ; p=0.011\right.$; GreenhouseGeisser-corrected), reflecting RT decreases from the 1st to 3rd presentation in the rewarded, but not in the neutral condition. Repetition alone showed only a trendwise effect on RTs $(p=0.086$, Greenhouse-Geisser-corrected; $p=0.073$, uncorrected).

\section{FUNCTIONAL MRI RESULTS}

\section{Effects of reward anticipation}

Irrespective of repetition and in line with previous studies (Knutson et al., 2001a,b; Wittmann et al., 2005; Schott et al., 2008; Krebs et al., 2009a,b) reward-predicting cues elicited activations of the mesolimbic reward system, particularly the VS/NAcc (Figure 2) and the SN/VTA at an uncorrected threshold of $p<0.001$ (Table 2). Activations in the NAcc survived a smallvolume correction for a combined anatomical and probabilistic ROI of the VS $(p<0.05$, FWE-corrected; see Materials and Methods for details on ROI generation).

\section{Effects of feedback}

When compared to neutral feedback, positive reward feedback was associated with activation of a cluster encompassing portions of the rostral anterior cingulate (rACC) and orbitofrontal cortex. No feedback-related NAcc activation was observed even at $p<0.05$,

Table 1 | Behavioral results.

\begin{tabular}{|c|c|c|c|}
\hline & 1st presentation & 2nd presentation & 3rd presentation \\
\hline \multicolumn{4}{|c|}{ NEUTRAL } \\
\hline$\%$ Hits & $62.6 \pm 14.92$ & $63.8 \pm 16.11$ & $65.4 \pm 12.61$ \\
\hline $\mathrm{RT}$ & $387 \pm 34.8$ & $384 \pm 33.5$ & $389 \pm 36.1$ \\
\hline \multicolumn{4}{|c|}{ REWARD } \\
\hline$\%$ Hits & $81.0 \pm 9.48$ & $83.1 \pm 9.89$ & $83.7 \pm 8.57$ \\
\hline $\mathrm{RT}$ & $371 \pm 33.0$ & $369 \pm 32.5$ & $368 \pm 30.4$ \\
\hline
\end{tabular}

Percentages and mean reaction times of successful target responses (hits, i.e., correct responses in the number comparison task within the individually adjusted time limit) are given, separated by condition (neutral vs. reward) and cue repetition (1st, 2nd, 3rd presentation). Group means $\pm S E$ are shown. Despite the nominally small RT decrease from 1 st to 3rd presentation, there was a significant interaction of reward and cue repetition $\left(F_{1.73,50.04}=5.27 ; p=0.011\right.$; Greenhouse-Geisser-corrected). 


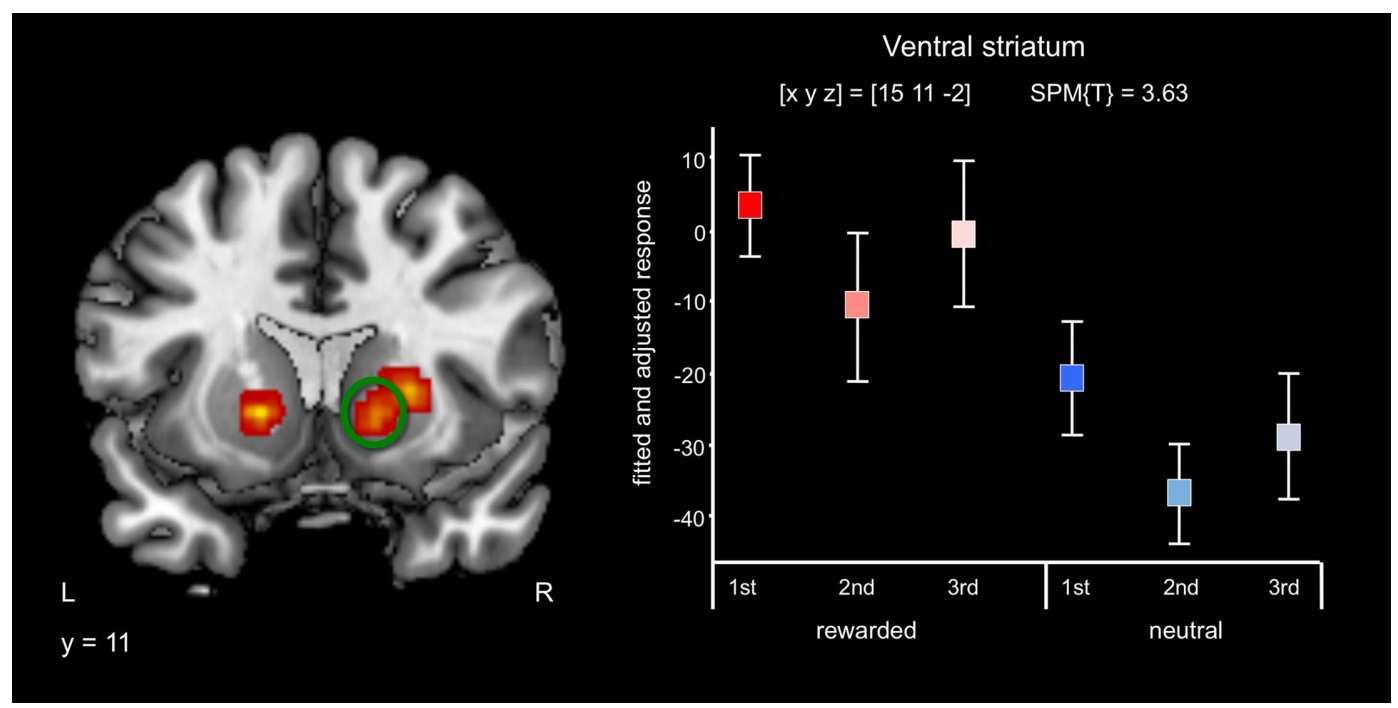

FIGURE 2 | Effects of reward anticipation. Reward cues compared to neutral cues were associated with bilateral activation of the ventral striatum/nucleus accumbens ( $p<0.05$, small-volume FWE-corrected).
Plots depict fitted and adjusted responses to cue pictures (1st, 2nd, and 3rd presentation, separated by rewarded and neutral condition) \pm SE.

Table 2 | Main effect of reward anticipation.

\begin{tabular}{|c|c|c|c|c|}
\hline Brain structure & $\boldsymbol{x}$ & $y$ & $z$ & SPM $\{T\}$ \\
\hline Left ventral striatum & -15 & 14 & -2 & $4.29 *$ \\
\hline \multirow[t]{3}{*}{ Right ventral striatum } & 24 & 11 & 4 & $4.05^{*}$ \\
\hline & 15 & 11 & -2 & $3.71^{*}$ \\
\hline & -21 & 2 & -2 & 3.38 \\
\hline Right dorsal striatum & 18 & 8 & 10 & 3.33 \\
\hline Midbrain/substantia nigra/subthalamic nucleus & 6 & -28 & -11 & 4.28 \\
\hline Right anterior cingulate, BA 32 & 12 & 32 & -5 & 4.24 \\
\hline Left thalamus & -3 & -22 & 1 & 3.85 \\
\hline Right thalamus & 9 & -16 & -2 & 3.87 \\
\hline
\end{tabular}

T-contrast comparing reward-predicting to neutral images, irrespective of repetition. Local maxima at $p<0.001$, uncorrected, are shown. ${ }^{*}$ These activations in the ventral striatum survived small-volume FWE correction for literature-based ROIs of the ventral striatum. BA, Brodmann area. All coordinates are given in MNI space (Montreal Neurological Institute).

uncorrected, a finding in line with previous results using similar tasks in young, healthy participants (Knutson et al., 2001a,b; Schott et al., 2007). Due to the low number of events, no analyses of fMRI responses to negative feedback are reported.

\section{Effect of repetition}

Irrespective of reward-predicting properties of the cue pictures, repeated presentation was associated with a widespread response decrease (i.e., RS) from 1st to 3 rd presentation. RS could be observed in secondary visual areas, in ventral visual stream structures (including fusiform and parahippocampal cortex) as well in MTL regions, including anterior and posterior hippocampus (Figure 3A, middle row) as well as portions of the amygdala (Figure 3A, bottom row). Furthermore, RS was also present in prefrontal cortical structures (Table 3). As previously reported for complex scene stimuli (Bunzeck et al., 2006), the local maximum of the RS contrast within the VS was observed in the PPA, as identified using a literature-based probabilistic ROI (Figure 3B; see Materials and Methods for details on ROI generation).

\section{Interaction of reward anticipation and repetition}

A T-contrast testing increased RS for reward-predicting cues (reward vs. neutral $\times 1$ st vs. 3 rd repetition; $p<0.001$, uncorrected) revealed the right anterior MTL as the only brain region, where repetition-related activation decreases were significantly stronger for rewarded than for neutral cues. To assess in detail which MTL regions showed an interaction of repetition and reward anticipation, we performed a detailed ROI analysis using ROIs from the WFU Pickatlas (Wake Forest University). The interaction contrast was thresholded at $p<0.005$, uncorrected, and significance levels and cluster sizes were computed for the hippocampus, the amygdala, and the parahippocampal cortex. In 


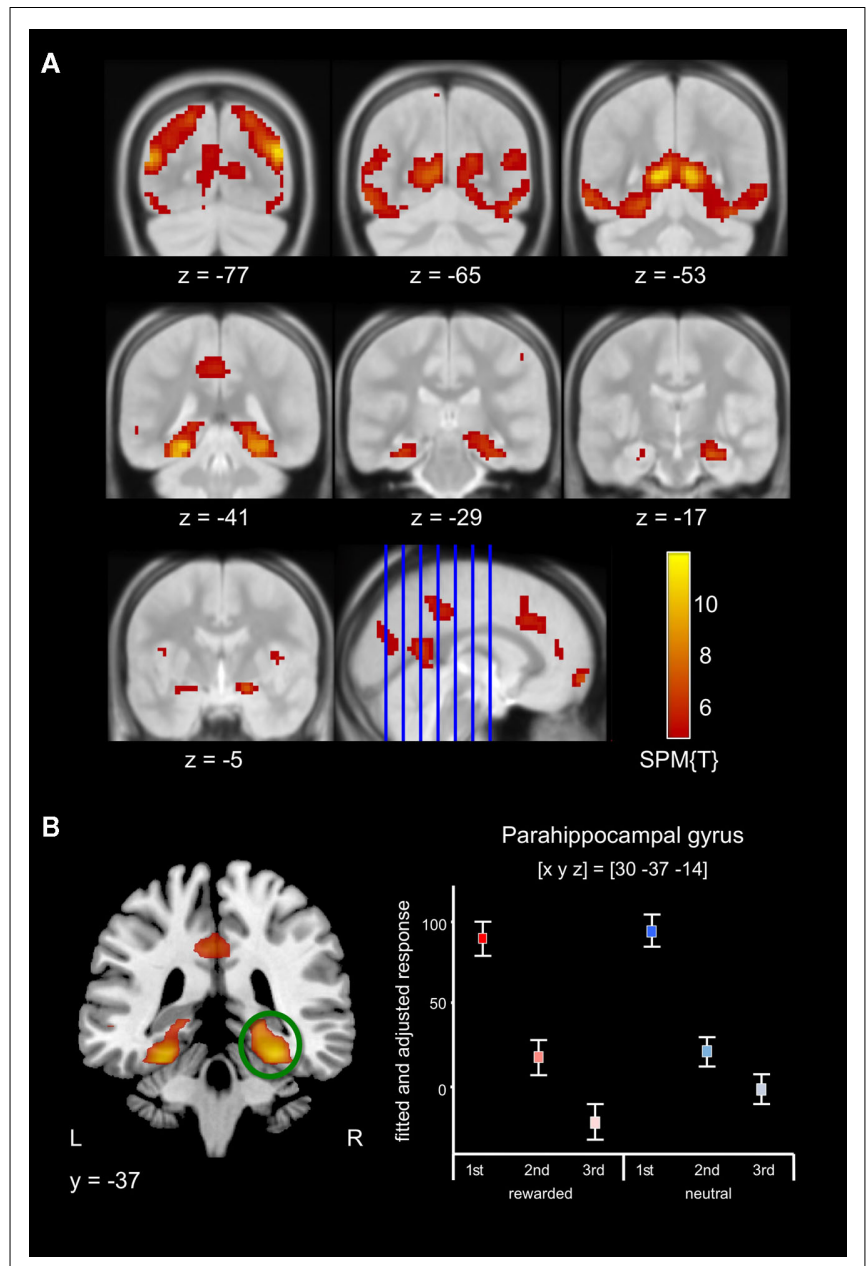

FIGURE 3 | Effect of repetition. (A) Scene stimuli were associated with response decreases from 1 st to 2 nd and from 2 nd to 3 rd presentation in an extensive network including secondary visual areas (top row) and in the medial temporal cortices, including the parahippocampal cortex and hippocampus (middle row), extending into the amygdala (bottom row). (B) Pronounced RS was observed in the posterior parahippocampal gyrus. Plots depict the fitted and adjusted responses to cue pictures $( \pm S E)$ separated by reward vs. neutral condition and repetition.

the right MTL, the local maximum and the largest portion of the cluster were located in the anterior hippocampus, and the activation survived a small-volume correction (FWE-corrected $p=0.001$ ) for the ROI (Figure 4; Table 4). A smaller portion of the cluster extended into the amygdala, where the activation also survived small-volume FWE correction, but showed no distinct local maximum. There was also a significant interaction of repetition and reward anticipation in the left anterior hippocampus ( $[x$ $y z]=[-24-13-14] ; p=0.026$, small-volume FWE-corrected; Figure 4). No significant FWE-correctable voxels were found in the left amygdala or in the parahippocampal cortex of either hemisphere.

\section{Functional connectivity results}

To assess potential modulations of stimulus-dependent functional connectivity between the hippocampus and other brain regions involved in stimulus perception and reward processing, we computed a PPI analysis of the repetition effects for rewardpredicting and neutral cues, with the right anterior hippocampus as seed region. In a voxel-wise linear contrast (PPI reward vs. PPI neutral), we observed specifically higher repetition-related functional coupling between the right anterior hippocampus and the posterior parahippocampal cortex and the medial OFC for rewardpredicting cue pictures when compared to neutral cue pictures (Figure 5). The cluster in the parahippocampal cortex largely overlapped a literature-based probabilistic ROI of the PPA (see above).

\section{DISCUSSION}

The present study demonstrates that reward anticipation specifically modulates hippocampal repetition responses, with greater RS for reward-predicting as compared to neutral stimuli. Furthermore, functional connectivity analysis suggests that the hippocampus might indeed act as an interface linking secondary stimulusreactive brain structures, such as the PPA and motivation-related structures, such as the OFC, during processing of repeatedly presented reward-predicting stimuli.

\section{INTERACTION OF REPETITION AND REWARD ANTICIPATION IN THE HIPPOCAMPUS}

As demonstrated previously, reward-predicting cues were associated with increased activation of the VS/NAcc (Knutson et al., 2001a,b; O'Doherty et al., 2002; Wittmann et al., 2005; Schott et al., 2007, 2008; for a review see Knutson and Cooper, 2005). Repetition-related response decreases were observed in secondary visual areas, including the PPA, in prefrontal cortical structures, and in the bilateral MTL. There was, on the other hand, no RS in the NAcc, where both novel and repeated reward cues were associated with comparable activation levels.

An interaction of reward-related motivational salience and repetition was observed reliably and specifically in the anterior hippocampus, particularly on the right side. In this region, only pictures signaling an upcoming reward were associated with robust RS. This response pattern is at odds with neural models of RS as a passive phenomenon like habituation, but favors models that consider RS an active learning mechanism that can be contextually modulated. Our results are well in line with the notion that stimulus responses might represent a prediction error, i.e., the difference between incoming excitatory bottom-up input ("evidence") and top-down modulatory signals reflecting previous information ("prediction"; Friston, 2005; Summerfield et al., 2008). With stimulus repetition, bottom-up and top-down information become increasingly congruent, resulting in an overall response decrease. It is conceivable that top-down modulatory input can be shaped as a function of previous information, such as stimulus salience, which, in our study, was defined upon the stimulus category. Indeed, Lisman and Grace (2005) have previously suggested that motivation-related hippocampal dopamine release might be a mechanism by which novel stimuli detected by the hippocampus are subsequently encoded.

Our observations are in good agreement with previously reported reward-related enhancement of hippocampusdependent long-term memory (Wittmann et al., 2005; Adcock 
Table 3 | Main effect of repetition (1st-3rd presentation).

\begin{tabular}{|c|c|c|c|c|}
\hline Brain structure & $x$ & $y$ & $z$ & SPM $\{T\}$ \\
\hline Left superior frontal gyrus, BA 9 & -15 & 53 & 31 & 6.96 \\
\hline Left middle frontal gyrus, BA 8 & -27 & 20 & 40 & 5.54 \\
\hline \multirow[t]{2}{*}{ Left inferior frontal gyrus, BA 44, 47} & -30 & 29 & -17 & 7.31 \\
\hline & -45 & 32 & 4 & 7.23 \\
\hline Right inferior frontal gyrus, BA 46, 47 & 48 & 35 & 13 & 6.53 \\
\hline Left medial frontal gyrus, BA 10 & 0 & 59 & -8 & 7.58 \\
\hline Left anterior cingulate, BA 32 & -3 & 44 & 10 & 5.60 \\
\hline \multirow[t]{2}{*}{ Left cingulate, BA 31, 32} & -3 & -37 & 40 & 6.68 \\
\hline & -15 & -43 & 37 & 5.97 \\
\hline Left parahippocampal gyrus/hippocampus, BA 36 & -24 & -43 & -14 & 10.69 \\
\hline \multirow[t]{2}{*}{ Right parahippocampal gyrus/hippocampus, BA 28, 36} & 30 & -37 & -14 & 10.49 \\
\hline & 24 & -19 & -20 & 8.35 \\
\hline \multirow[t]{2}{*}{ Left middle temporal gyrus, BA 37, 39} & -45 & -76 & 16 & 10.07 \\
\hline & -57 & -55 & -11 & 8.07 \\
\hline Right middle temporal gyrus, BA 39 & 42 & -76 & 22 & 11.12 \\
\hline Left fusiform gyrus, BA 19 & -48 & -70 & -14 & 8.60 \\
\hline Right fusiform gyrus, BA 37 & 48 & -61 & -14 & 7.95 \\
\hline Left insula, BA 13 & -39 & -4 & 13 & 5.52 \\
\hline Right insula & 39 & -4 & 7 & 6.10 \\
\hline
\end{tabular}

Local maxima are displayed at $p<0.05$, whole-brain FWE-corrected. All coordinates are given in MNI space (Montreal Neurological Institute).

et al., 2006; Krebs et al., 2009a) and reward cues show particularly enhanced recollection, which is, unlike familiarity thought to be critically dependent on the hippocampus (Düzel et al., 2001; Yonelinas et al., 2002). A recent study has demonstrated that neuronal populations in hippocampus and in adjacent cortical structures can adaptively code both reward magnitude and stimulus novelty as a function of contextual predictions (Bunzeck et al., 2010). The present study extends this finding by demonstrating that hippocampal coding of novelty can also be modulated by salience of a stimulus as indexed by its reward-predicting property.

Unlike the hippocampus, the NAcc showed no clear interaction between reward anticipation and repetition, with novel and repeated reward cues eliciting similarly strong reward anticipation responses. In the framework of the hippocampal-VTA loop (Lisman and Grace, 2005), this might suggest that, once the hippocampus has transmitted information regarding a novel salient stimulus to the NAcc, hippocampus-independent mechanisms might subsequently trigger a NAcc response to reward on subsequent presentations of the stimulus. Alternatively, and perhaps more likely, a reduced but more specific signal from the hippocampus might be as effective in eliciting a NAcc response as the initial response to the novel stimulus.
It should be noted that repetition lags were short, with all three presentations of each stimulus occurring within 18 trials. Because such short repetition lags are associated with stronger RS than longer lags (Brozinsky et al., 2005), we cannot generalize our findings to reward-predicting stimuli repeated after longer delays. A recent study, however, suggests that novelty/familiarity interactions in the hippocampus can also be observed after longer delays (Bunzeck et al., 2010).

\section{HIPPOCAMPAL-CORTICAL FUNCTIONAL CONNECTIVITY}

Because the hippocampus was the only structure to show reliable RS specifically for reward-predicting pictures, we had hypothesized that enhanced activity-dependent modulation functional connectivity between the hippocampus and both stimulus-related and reward-related brain structures might provide a mechanism for hippocampus-dependent linking of reward signals and higherlevel stimulus perception. In line with this hypothesis, our PPI revealed a stronger functional connectivity of the hippocampus with the PPA and the OFC during repetition of reward cues, but not neutral cues (Figure 5).

Previous studies of reward-related functional connectivity have largely focused on the NAcc (Menon and Levitin, 2005; Schott 


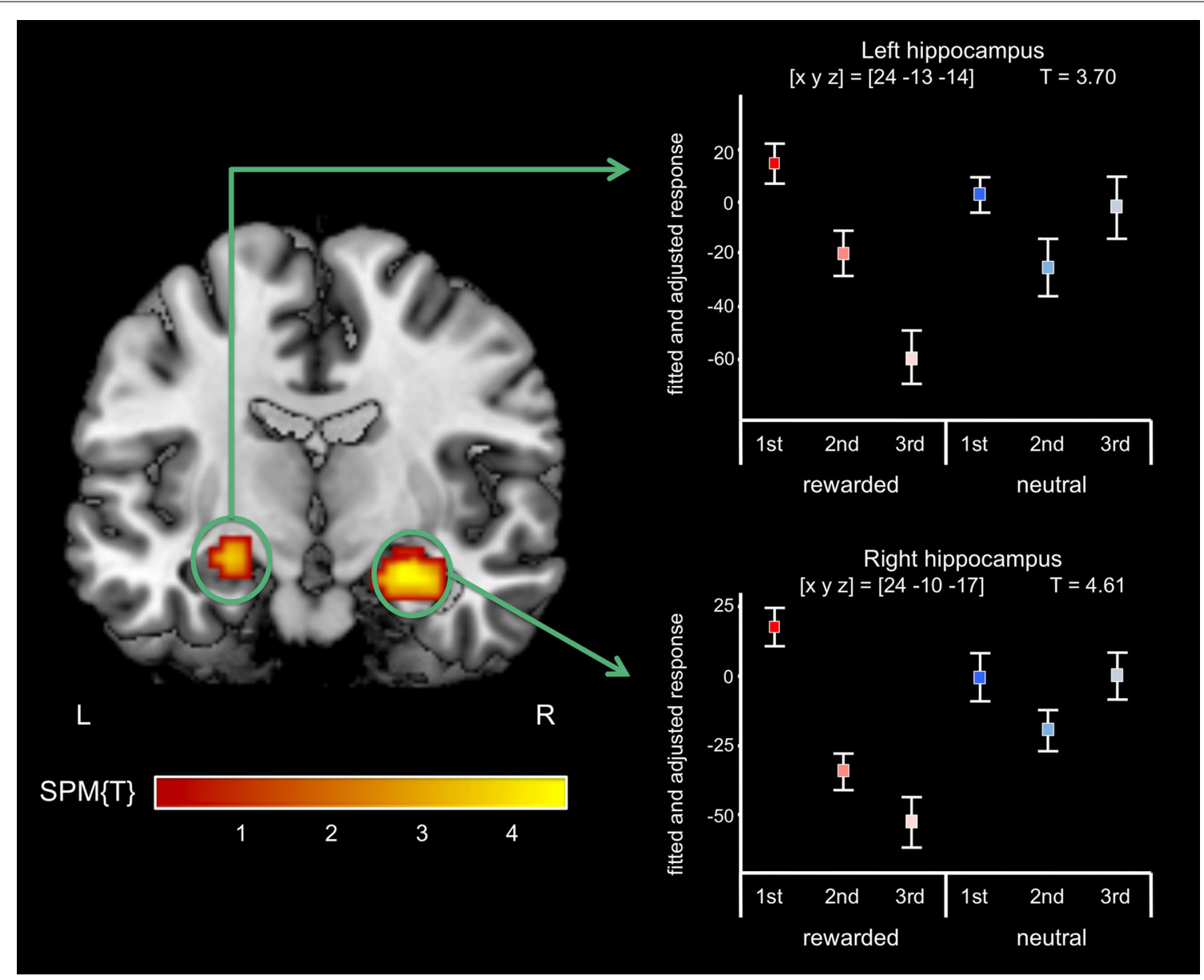

FIGURE 4 | Interaction of reward anticipation and cue repetition in the anterior hippocampus. In the right anterior hippocampus, repetition suppression was primarily observed for reward cues relative to neutral cues $(p<0.05$, small-volume FWE-corrected). Plots depict fitted and adjusted responses to cue pictures (1st, 2nd, and 3rd presentation, separated by rewarded and neutral condition) $\pm \mathrm{SE}$.

Table 4 | Interaction of repetition and reward in the MTL.

\begin{tabular}{|c|c|c|c|c|c|c|}
\hline & Cluster size $\left(\mathrm{mm}^{3}\right)$ & $x$ & $y$ & $z$ & Max. SPM $\{T\}$ & $p$ (FWE-corrected) \\
\hline \multicolumn{7}{|c|}{ HIPPOCAMPUS } \\
\hline Left & 297 & -24 & -13 & -14 & 3.70 & $0.026^{*}$ \\
\hline Right & 972 & 24 & -10 & -17 & 4.61 & $0.001 *$ \\
\hline \multicolumn{7}{|c|}{ PARAHIPPOCAMPAL CORTEX } \\
\hline Left & 0 & n/a & & & n/a & n/a \\
\hline Right & 81 & 21 & -10 & -23 & 3.05 & 0.180 \\
\hline \multicolumn{7}{|c|}{ AMYGDALA } \\
\hline Left & 0 & $\mathrm{n} / \mathrm{a}$ & & & $\mathrm{n} / \mathrm{a}$ & $\mathrm{n} / \mathrm{a}$ \\
\hline Right & 189 & 24 & -7 & -17 & 4.24 & $0.001 *$ \\
\hline
\end{tabular}

Results from ROI-based analyses are shown. ${ }^{*} p<0.05$, small-volume FWE-corrected for ROIs derived from the WFU Pickatlas.

et al., 2007; Camara et al., 2008; Schmack et al., 2008), which shows extensive reward-related functional connectivity with distant brain regions, including the midbrain, the rACC/MPFC, the hippocampus, and the amygdala. While the hippocampus has relatively consistently been found to functionally interact with the midbrain and NAcc (Adcock et al., 2006; Callan and Schweighofer,
2008; Camara et al., 2008), it has thus far rarely been the focus of studies investigating reward-related functional connectivity. A recent study investigating memory encoding of faces, however, has demonstrated that functional connectivity of the OFC correlates with hippocampal activity during encoding of attractive, but not unattractive faces (Tsukiura and Cabeza, 2011), and processing of 


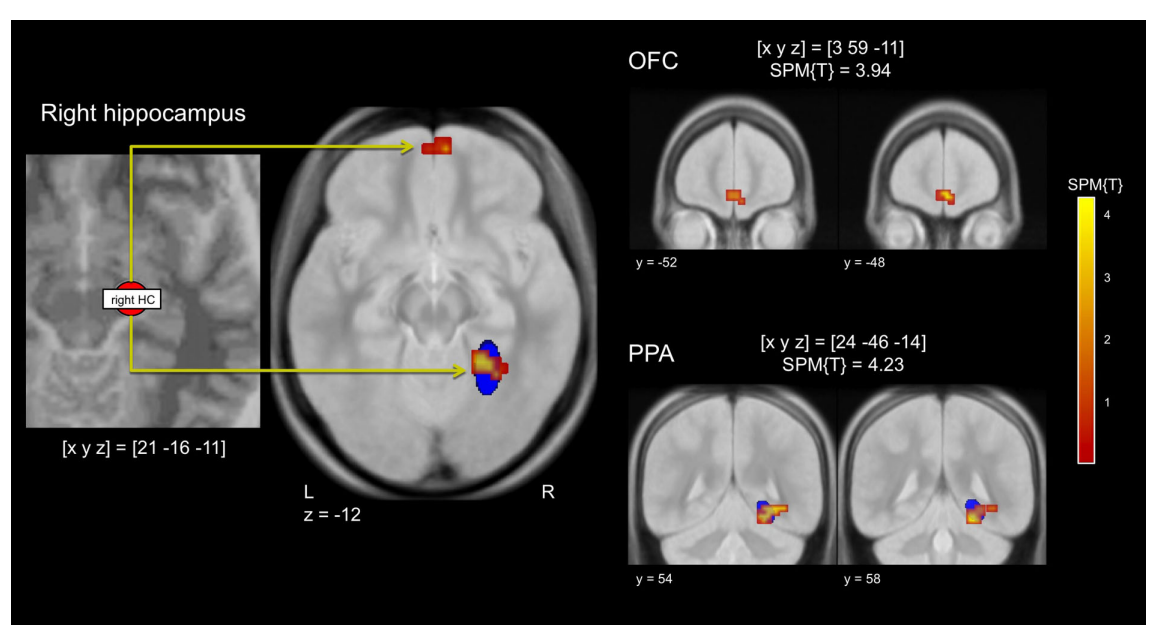

FIGURE 5 | Stimulus-dependent functional connectivity of the hippocampus during repetition of reward cues relative to neutral cues. Left panel: Representative seed region in the right anterior hippocampus. Middle panel: The right hippocampus showed increased functional connectivity with the right parahippocampal place area (PPA) and with the medial orbitofrontal cortex (OFC). Right panel: Representative coronal sections depicting the extent of the functional connectivity increases in the medial OFC (top) and in the PPA (bottom). The blue ellipsoid denotes the extent of a literature-based ROI of the PPA (see Materials and Methods for details). $p<0.001$, uncorrected; minimum cluster size $=10$ adjacent voxels. attractive faces has been demonstrated to engage the mesolimbic reward system (Bray and O'Doherty, 2007). The study by Tsukiura and Cabeza reported subsequent memory effects in the fusiform face area (FFA), but did not investigate potential activitydependent connectivity changes of the FFA and hippocampus. It should be noted here that our PPI analysis, while providing information about activity-dependent functional connectivity, does not allow to make inferences about the directionality of the functional interactions, and we ca therefore not conclude whether the hippocampus primarily modulates activity in the OFC or PPA or vice versa. In the light of our current results, however, we do suggest that the hippocampus might act as an interface linking brain regions involved in higher-level stimulus perception to those primarily engaged in motivational processing.

\section{REFERENCES}

Abler, B., Erk, S., and Walter, H. (2007). Human reward system activation is modulated by a single dose of olanzapine in healthy subjects in an event-related, double-blind, placebo-controlled fMRI study. Psychopharmacology (Berl.) 191, 823-833.

Abler, B., Walter, H., Erk, S., Kammerer, H., and Spitzer, M. (2006). Prediction error as a linear function of reward probability is coded in human nucleus accumbens. Neuroimage 31, 790-795.

Adcock, R. A., Thangavel, A., WhitfieldGabrieli, S., Knutson, B., and Gabrieli, J. D. (2006). Rewardmotivated learning: mesolimbic activation precedes memory formation. Neuron 50, 507-517.

Bray, S., and O'Doherty, J. (2007). Neural coding of reward-prediction error signals during classical conditioning with attractive faces. $J$. Neurophysiol. 97, 3036-3045. and Ashburner, J. (2001). Spatial normalization of brain images with focal lesions using cost function masking. Neuroimage 14, 486-500.

Brozinsky, C. J., Yonelinas, A. P., Kroll, N. E., and Ranganath, C. (2005). Lag-sensitive repetition suppression effects in the anterior parahippocampal gyrus. Hippocampus 15, 557-561.

Bunzeck, N., Dayan, P., Dolan, R. J., and Düzel, E. (2010). A common mechanism for adaptive scaling of reward and novelty. Hum. Brain Mapp. 31, 1380-1394.

Bunzeck, N., Schütze, H., and Düzel, E. (2006). Category-specific organization of prefrontal responsefacilitation during priming. Neuropsychologia 44, 1765-1776.
Brett, M., Leff, A. P., Rorden, C.,

\section{CONCLUSION}

Our results demonstrate that the reward-predicting properties of complex scene stimuli selectively modulate hippocampal repetition responses, with a RS primarily for reward-predicting stimuli. Functional connectivity analysis further suggests that the hippocampus might act as an interface linking stimulusresponsive brain structures with those related to motivational processing.

\section{ACKNOWLEDGMENTS}

Supported by the Deutsche Forschungsgemeinschaft (DFG SFB 779, TP A7/A8 and DFG RI 1847), and the State of SaxonyAnhalt. We thank Kerstin Möhring, Ilona Wiedenhöft, and Claus Tempelmann for assistance with MRI acquisition and analysis.

Callan, D. E., and Schweighofer, N. (2008). Positive and negative modulation of word learning by reward anticipation. Hum. Brain Mapp. 29, 237-249.

Camara, E., Rodriguez-Fornells, A., and Munte, T. F. (2008). Functional connectivity of reward processing in the brain. Front. Hum. Neurosci. 2:19. doi:10.3389/neuro.09.019.2008

Camara, E., Rodriguez-Fornells, A., and Munte, T. F. (2010). Microstructural brain differences predict functional hemodynamic responses in a reward processing task. J. Neurosci. 30, 11398-11402.

D’Ardenne, K., McClure, S. M., Nystrom, L. E., and Cohen, J. D. (2008). BOLD responses reflecting dopaminergic signals in the human ventral tegmental area. Science 319, 1264-1267.

Das, P., Kemp, A. H., Liddell, B. J., Brown, K. J., Olivieri, G., Peduto,
A., Gordon, E., and Williams, L. M. (2005). Pathways for fear perception: modulation of amygdala activity by thalamo-cortical systems. Neuroimage 26, 141-148.

Desimone, R. (1996). Neural mechanisms for visual memory and their role in attention. Proc. Natl. Acad. Sci. U.S.A. 93, 13494-13499.

Düzel, E., Bunzeck, N., Guitart-Masip, M., and Düzel, S. (2010). NOveltyrelated motivation of anticipation and exploration by dopamine (NOMAD): implications for healthy aging. Neurosci. Biobehav. Rev. 34, 660-669.

Düzel, E., Vargha-Khadem, F., Heinze, H. J., and Mishkin, M. (2001). Brain activity evidence for recognition without recollection after early hippocampal damage. Proc. Natl. Acad. Sci. U.S.A. 98, 8101-8106. 
Eickhoff, S. B., Heim, S., Zilles, K., and Amunts, K. (2006). Testing anatomically specified hypotheses in functional imaging using cytoarchitectonic maps. Neuroimage 32, 570-582.

Eickhoff, S. B., Stephan, K. E., Mohlberg, H., Grefkes, C., Fink, G. R., Amunts, K., and Zilles, K. (2005). A new SPM toolbox for combining probabilistic cytoarchitectonic maps and functional imaging data. Neuroimage 25, 1325-1335.

Epstein, R. A., and Higgins, J. S. (2007). Differential parahippocampal and retrosplenial involvement in three types of visual scene recognition. Cereb. Cortex 17, 1680-1693.

Epstein, R. A., Higgins, J. S., Jablonski, K., and Feiler, A. M. (2007). Visual scene processing in familiar and unfamiliar environments. J. Neurophysiol. 97, 3670-3683.

Friston, K. (2005). A theory of cortical responses. Philos. Trans. R. Soc. Lond. B Biol. Sci. 360, 815-836.

Friston, K. J., Buechel, C., Fink, G. R., Morris, J., Rolls, E., and Dolan, R. J. (1997). Psychophysiological and modulatory interactions in neuroimaging. Neuroimage 6, 218-229.

Gitelman, D. R., Penny, W. D., Ashburner, J., and Friston, K. J. (2003). Modeling regional and psychophysiologic interactions in fMRI: the importance of hemodynamic deconvolution. Neuroimage 19, 200-207.

Grill-Spector, K., Henson, R., and Martin, A. (2006). Repetition and the brain: neural models of stimulus-specific effects. Trends Cogn. Sci. (Regul. Ed.) 10, 14-23.

Hariri, A. R., Brown, S. M., Williamson, D. E., Flory, J. D., De Wit, H., and Manuck, S. B. (2006). Preference for immediate over delayed rewards is associated with magnitude of ventral striatal activity. J. Neurosci. 26, 13213-13217.

Henderson, J. M., Larson, C. L., and Zhu, D. C. (2008). Full scenes produce more activation than close-up scenes and scene-diagnostic objects in parahippocampal and retrosplenial cortex: an fMRI study. Brain Cogn. 66, 40-49.

Henson, R. N., and Mouchlianitis, E. (2007). Effect of spatial attention on stimulus-specific haemodynamic repetition effects. Neuroimage 35, 1317-1329.

Hinrichs, H., Scholz, M., Tempelmann, C., Woldorff, M. G., Dale, A. M., and Heinze, H. J. (2000). Deconvolution of event-related fMRI responses in fast-rate experimental designs: tracking amplitude variations. J. Cogn. Neurosci. 12(Suppl. 2), 76-89.

Horner, A. J., and Andrews, T. J. (2009). Linearity of the fMRI response in category-selective regions of human visual cortex. Hum. Brain Mapp. 30, 2628-2640

Ishai, A., Pessoa, L., Bikle, P. C., and Ungerleider, L. G. (2004). Repetition suppression of faces is modulated by emotion. Proc. Natl. Acad. Sci. U.S.A. 101, 9827-9832.

James, T. W., and Gauthier, I. (2006). Repetition-induced changes in BOLD response reflect accumulation of neural activity. Hum. Brain Mapp. 27, 37-46.

Juckel, G., Schlagenhauf, F., Koslowski, M., Wüstenberg, T., Villringer, A., Knutson, B., Wrase, J., and Heinz, A. (2006). Dysfunction of ventral striatal reward prediction in schizophrenia. Neuroimage 29, 409-416.

Knutson, B., Adams, C. M., Fong, G. W., and Hommer, D. (2001a). Anticipation of increasing monetary reward selectively recruits nucleus accumbens. J. Neurosci. 21, RC159.

Knutson, B., Fong, G. W., Adams, C. M., Varner, J. L., and Hommer, D. (2001b). Dissociation of reward anticipation and outcome with event-related fMRI. Neuroreport 12, 3683-3687.

Knutson, B., and Cooper, J. C. (2005). Functional magnetic resonance imaging of reward prediction. Curr. Opin. Neurol. 18, 411-417.

Krebs, R. M., Schott, B. H., and Düzel, E. (2009a). Personality traits are differentially associated with patterns of reward and novelty processing in the human substantia nigra/ventral tegmental area. Biol. Psychiatry 65, 103-110.

Krebs, R. M., Schott, B. H., Schütze, H., and Düzel, E. (2009b). The novelty exploration bonus and its attentional modulation. Neuropsychologia 47, 2272-2281.

Lisman, J. E., and Grace, A. A. (2005). The hippocampal-VTA loop: controlling the entry of information into long-term memory. Neuron 46, 703-713.
McClure, S. M., Daw, N. D., and Montague, P. R. (2003). A computational substrate for incentive salience. Trends Neurosci. 26, 423-428.

Menon, V., and Levitin, D. J. (2005). The rewards of music listening: response and physiological connectivity of the mesolimbic system. Neuroimage 28, 175-184.

Nielsen, F. A., and Hansen, L. K. (2002). Modeling of activation data in the BrainMap database: detection of outliers. Hum. Brain Mapp. 15, 146-156.

O’Doherty, J., Dayan, P., Schultz, J., Deichmann, R., Friston, K., and Dolan, R. J. (2004). Dissociable roles of ventral and dorsal striatum in instrumental conditioning. Science 304, 452-454.

O’Doherty, J. P., Deichmann, R., Critchley, H. D., and Dolan, R. J. (2002). Neural responses during anticipation of a primary taste reward. $\mathrm{Neu}$ ron $33,815-826$.

Park, S., Chun, M. M., and Johnson, M. K. (2009). Refreshing and integrating visual scenes in sceneselective cortex. J. Cogn. Neurosci. 22, 2813-2822.

Park, S., Intraub, H., Yi, D. J., Widders, D., and Chun, M. M. (2007). Beyond the edges of a view: boundary extension in human sceneselective visual cortex. Neuron 54, 335-342.

Peelen, M. V., and Downing, P. E. (2005). Within-subject reproducibility of category-specific visual activation with functional MRI. Hum. Brain Mapp. 25, 402-408.

Ranganath, C., and Rainer, G. (2003) Neural mechanisms for detecting and remembering novel events. Nat. Rev. Neurosci. 4 193-202.

Scheres, A., Milham, M. P., Knutson, B. and Castellanos, F. X. (2007). Ventral striatal hyporesponsiveness during reward anticipation in attentiondeficit/hyperactivity disorder. Biol. Psychiatry 61, 720-724.

Schmack, K., Schlagenhauf, F., Sterzer, P., Wrase, J., Beck, A., Dembler, T., Kalus, P., Puls, I., Sander, T., Heinz, A., and Gallinat, J. (2008). CatecholO-methyltransferase val158met genotype influences neural processing of reward anticipation. Neuroimage 42, 1631-1638.

Schott, B. H., Minuzzi, L., Krebs, R. M., Elmenhorst, D., Lang, M., Winz, O. H., Seidenbecher, C. I., Coenen, H. H., Heinze, H. J., Zilles, K., Düzel, E., and Bauer, A.
(2008). Mesolimbic functional magnetic resonance imaging activations during reward anticipation correlate with reward-related ventral striatal dopamine release. J. Neurosci. 28, 14311-14319.

Schott, B. H., Niehaus, L., Wittmann, B. C., Schütze, H., Seidenbecher, C. I., Heinze, H. J., and Düzel, E. (2007). Ageing and early-stage Parkinson's disease affect separable neural mechanisms of mesolimbic reward processing. Brain 130, 2412-2424.

Schubert, R., Ritter, P., Wüstenberg, T., Preuschhof, C., Curio, G., Sommer, W., and Villringer, A. (2008). Spatial attention related SEP amplitude modulations covary with BOLD signal in S1- a simultaneous EEG fMRI study. Cereb. Cortex 18, 2686-2700.

Schultz, W. (1997). Dopamine neurons and their role in reward mechanisms. Curr. Opin. Neurobiol. 7, 191-197.

Summerfield, C., Trittschuh, E. H., Monti, J. M., Mesulam, M. M., and Egner, T. (2008). Neural repetition suppression reflects fulfilled perceptual expectations. Nat. Neurosci. 11, 1004-1006.

Tsukiura, T., and Cabeza, R. (2011). Remembering beauty: roles of orbitofrontal and hippocampal regions in successful memory encoding of attractive faces. Neuroimage 54, 653-660.

Tulving, E., Markowitsch, H. J., Craik, F. E., Habib, R., and Houle, S. (1996). Novelty and familiarity activations in PET studies of memory encoding and retrieval. Cereb. Cortex 6, 71-79.

Turkeltaub, P. E., Eden, G. F., Jones, K. M., and Zeffiro, T. A. (2002). Meta-analysis of the functional neuroanatomy of single-word reading: method and validation. Neuroimage 16, 765-780.

Vuilleumier, P., Schwartz, S., Duhoux, S., Dolan, R. J., and Driver, J. (2005). Selective attention modulates neural substrates of repetition priming and "implicit” visual memory: suppressions and enhancements revealed by FMRI. J. Cogn. Neurosci. 17, 1245-1260.

Wittmann, B. C., Schott, B. H., Guderian, S., Frey, J. U., Heinze, H. J., and Düzel, E. (2005). Reward-related FMRI activation of dopaminergic midbrain is associated with enhanced hippocampus-dependent long-term memory formation. $\mathrm{Neu}$ ron 45, 459-467. 
$\mathrm{Xu}$, Y., Turk-Browne, N. B., and Chun, M. M. (2007). Dissociating task performance from fMRI repetition attenuation in ventral visual cortex. J. Neurosci. 27, 5981-5985.

Yonelinas, A. P., Kroll, N. E., Quamme,

J. R., Lazzara, M. M., Sauve, M.

J., Widaman, K. F., and Knight,

R. T. (2002). Effects of extensive temporal lobe damage or mild hypoxia on recollection and familiarity. Nat. Neurosci. 5, 1236-1241.

Conflict of Interest Statement: The authors declare that the research was conducted in the absence of any commercial or financial relationships that could be construed as a potential conflict of interest.
Received: 25 June 2011; accepted: 02 November 2011; published online: 17 November 2011.

Citation: Zweynert S, Pade JP, Wüstenberg $T$, Sterzer $P$, Walter H, Seidenbecher CI, Richardson-Klavehn A, Düzel E and Schott BH (2011) Motivational salience modulates hippocampal repetition suppression and functional connectivity in humans. Front. Hum. Neurosci. 5:144. doi: 10.3389/fnhum.2011.00144
Copyright (C) 2011 Zweynert, Pade, Wüstenberg, Sterzer, Walter, Seidenbecher, Richardson-Klavehn, Düzel and Schott. This is an open-access article subject to a non-exclusive license between the authors and Frontiers Media $S A$, which permits use, distribution and reproduction in other forums, provided the original authors and source are credited and other Frontiers conditions are complied with. 